\title{
Decisions of Green Product Development and Pricing for a Dual Channel Supply Chain
}

\author{
Jianhua Wang ${ }^{1, a^{*}}$, Xianfeng Huang ${ }^{1, b}$, Ting Cao ${ }^{1, c}$ and Yue Liü, \\ ${ }^{1}$ Management School, Jiangsu University, Zhenjiang, 212013, China \\ ajiannywang@163.com, bhuangxianfeng@ujs.edu.cn, ${ }^{\circ} 642607727 @ q q . c o m$, \\ d1911817377@qq.com
}

\begin{abstract}
This paper focuses on the decisions of the green product development and sell pricing in a supply chain which has an offline channel and an online channel simultaneously. Considering some important customer's attributes, such as channel loyalty, price sensitivity and green preference, the optimal green degrees and the selling prices of the developing product are deduced by the Stackelberg game models for the centralized decision case and the decentralized decision case respectively, and the impact of customers' channel loyalty on the decision variables and the system performances of the supply chain are explored thoroughly and deeply. The research shows some interesting conclusions: (1) in the decentralized case, customer's online shopping preference has positive impacts on the product green degree, the wholesale price and the online selling quantity, has negative impacts on the selling price, the offline retailing quantity and the offline retailer's profit, and has positive impacts on the manufacturer's profit when the loyalty is small, but has negative impacts on the manufacturer's profit when the loyalty is bigger than a specific value. (2) in the centralized case, the optimal green degree of product and the whole supply chain profit are bigger than those in the decentralized case.
\end{abstract}

Keywords: Dual channel; Supply chain; Product green degree; Selling price; Online shopping preference

\section{双渠道供应链绿色产品开发与销售定价决策}

\author{
王建华，黄贤凤，曹婷，刘越 \\ （江苏大学管理学院，江苏 镇江 212013 )
}

摘要: 针对线上网络直销和线下零售商分销并存的双渠道供应链绿色产品开发与销售定价问题, 考虑顾客对渠道的偏好、 价格敏感性对产品绿色属性的偏好, 构建集中决策和制造商主导下分散决策的数学模型, 推导出两种决策情形下的最优销售 定价和产品绿色属性。研究结果表明: 分散决策下顾客的网购偏好对产品绿色属性、批发价格、网络直销销售量具有正向影 响，对零售价格、零售销售量以及零售商利润具有负向影响，而对制造商利润具有先正向后负向的影响; 集中决策下的产品 绿色属性总要高于分散决策下产品绿色属性, 集中决策下供应链利润总要高于分散决策下供应链利润。

关键词: 双渠道; 供应链; 产品绿色属性; 销售定价; 网购偏好;

引言

随着生活水平的提高和环保意识的增强, 人们对绿色产品的需求日益增多, 考虑资源消耗和环境影响 的绿色供应链模式开始受到关注 ${ }^{[1]}$ 。Berman（1999）提出供应链管理中要重视环境因素, 并提出对绿色供 应链的绩效考核要包括资源回收率、核心回报率、废物比、生态有效性等新的指标 ${ }^{[2]}$ 。Wu (2015) 研究不 确定市场环境下绿色供应链决策过程中的重要影响因素 ${ }^{[3]}$ 。Zohal (2016) 等对绿色供应链网络优化问题进 行建模和优化算法设计 ${ }^{[4]}$ 。近来, 相关学者开始研究产品绿色属性对供应链的影响, Grosh (2015) 对顾客 具有绿色敏感性供应链中绿色产品开发成本分担问题进行研究 ${ }^{[5]}$ 。随着电子商务的发展, 很多制造企业不 仅采取零售商渠道销售, 而且也通过网络直销的方式进行产品销售, 从而使得供应链呈现出双渠道的运营 
模式。Takahashi（2011）研究具有生产准备时间的双渠道供应链生产和配送优化问题, 以实现在最低库 存和缺货成本下运作, 并达成两个渠道的协调并存 ${ }^{[6]}$; Panda（2015）研究产品单位成本同批量成负相关关 系的供应链如何进行产品定价和补货决策, 以实现网络直销和零售渠道之间的利润协调 ${ }^{[7]}$; Li (2016) 研 究绿色供应链开发设计绿色产品的成本在一定范围内, 制造商网络直销和零售商渠道销售可以很好地协调 [8]。

本文在相关研究基础上，对由一个制造商和一个零售商组成的两级双渠道供应链在顾客具有绿色偏好 情况下的产品绿色属性开发和销售定价决策进行研究, 采取 Stackelberg 博弯获得集中决策和分散决策下 供应链的最优绿色产品开发策略和市场销售定价, 并分析顾客网购偏好对系统决策变量和绩效指标的影 响, 以及供应链开发绿色产品和实现双渠道运作的必要条件, 再通过算例详细地研究不同参数下供应链决 策变量和绩效指标的变化趋势和规律, 最后给出一些有价值的管理建议。

\section{1. 问题描述及数学建模}

考虑一个具有线上网络直销和线下零售并存的双渠道供应链。制造商负责设计和制造最终的产品，产 品制造结束后, 一部分产品通过网络平台直接销售给最终客户，另一部分产品则以价格 $w$ 批发给零售商, 然后零售商销售给最终客户。在当前低碳环保理念越来越广为接受的环境下，客户对产品的环保特性将有 一定的敏感性, 当产品绿色特征 $g$ 越明显时, 客户购买该类产品的数量越多, 反之则购买数量越少。同时 客户对产品价格 $p$ 具有敏感性, 当产品销售价格越高时, 顾客购买该类产品的数量越少, 反之则越多。为 了最大化供应链或自身的利润, 供应链成员需要确定拟开发产品的绿色属性 $g$ 以及最后向市场销售的零售 价格 $p$ 。

商业系统主要决策目标是利润最大化, 本文研究的供应链绿色产品开发和销售定价决策同样以利润最 大为目标。在进行利润模型构建之前, 先确定市场需求量模型。借鉴 Ghosh 的研究 ${ }^{[5]}$, 假设顾客需求同产 品定价以及产品绿色属性之间具有线性函数关系, 同时为了降低渠道销售冲突, 网络直销和零售价格相同, 据此设定两个渠道的客户需求量函数如式 (1) 和 (2)。

$$
\begin{aligned}
& D_{i}=\alpha d-\beta p+\gamma_{i} g \\
& D_{r}=(1-\alpha) d-\beta p+\gamma_{r} g
\end{aligned}
$$

式中: $D_{i}$ 和 $D_{r}$ 分别是网络直销量和零售店分销量; $d$ 为市场对该绿色产品的基本需求量； $\alpha$ 为客户 的网络购物偏好, $1-\alpha$ 为客户对线下零售购物的偏好, $0 \leq \alpha \leq 1 ; \beta$ 为顾客对产品价格的敏感系数; $\gamma_{i}$ 和 $\gamma_{r}$ 分别为顾客通过网络直销和零售分销购买产品时对产品绿色属性的偏好程度, 因为客户可以在零售店中 接触到产品的具体形态和特性, 所以假定 $0 \leq \gamma_{i}<\gamma_{r}$ 。

由于市场需求不小于零，制造商给予零售商的批发价格 $w$ 不会小于制造商的制造成本 $c$, 市场零售价 格 $p$ 不会小于零售商的批发价格 $w$, 因此有不等式 (3) 成立。

$$
c \leq w \leq p \leq \min \left(\frac{\alpha d+\gamma_{i} g}{\beta}, \frac{(1-\alpha) d+\gamma_{r} g}{\beta}\right)
$$

本文假定制造商生产的绿色产品对其其他传统产品的市场销量没有影响, 但是绿色产品需要进行创新 
型开发或资源投入, 即产品绿色属性需要额外的成本方能实现。借鉴Ghosh（2012）的研究 ${ }^{[15]}$, 设定成本 投入与产品绿色属性之间满足二次函数关系, 具体如式 (4) 所示, 式中 $\eta$ 为产品绿色属性成本系数。

$$
C(g)=\eta g^{2} / 2
$$

通过以上的分析, 可以获得制造商利润 $\pi_{i}$ 和零售商 $\pi_{r}$ 利润函数如下:

$$
\begin{aligned}
& \pi_{i}=(w-c)\left[(1-\alpha) d-\beta p+\gamma_{r} g\right]+(p-c)\left(\alpha d-\beta p+\gamma_{i} g\right)-0.5 \eta g^{2} \\
& \pi_{r}=(p-w)\left[(1-\alpha) d-\beta p+\gamma_{r} g\right]
\end{aligned}
$$

\section{2. 模型求解与分析}

\section{1 集中决策下的模型求解}

在集中决策情形下, 制造商和零售商作为一个整体进行绿色产品开发和销售定价决策, 以获得最大的 供应链利润。令 $\pi^{C}$ 为集中决策的供应链整体利润, 根据（5）和（6）可得其表达式如下:

$$
\pi^{c}=(p-c)\left[d-2 \beta p+\left(\gamma_{i}+\gamma_{r}\right) g\right]-0.5 \eta g^{2}
$$

由于供应链整体利润 $\pi^{C}$ 对 $p$ 和 $g$ 均为二阶可导, 且对 $p$ 的二阶偏导数为 $-4 \beta<0$, 对 $g$ 的二阶偏导数 为 $-\eta<0$, 因此该函数能够获得最大值的充要条件只需式 (8) 成立即可。

$$
4 \beta \eta>\left(\gamma_{i}+\gamma_{r}\right)^{2}
$$

在当前市场环境下, 顾客会追求产品的绿色属性, 但是在产品绿色属性和价格之间, 价格的影响效应 一般要大于产品绿色属性的影响效应, 即参数 $\beta$ 通常要比参数 $\gamma_{i}$ 和 $\gamma_{r}$ 大; 同时产品开发的成本相对较高, 绿色属性价格系数 $\eta$ 通常要比参数 $\gamma_{i}$ 和 $\gamma_{r}$ 大。因此式 (8) 在绝大多数情况下都会成立, 本研究假设该条 件总是成立。

当参数满足式 (8) 时, 集中决策可以获得最大供应链利润, 此时 $p$ 和 $g$ 最优取值如式 (9) 和 (10) 所示。

$$
\begin{aligned}
& p_{C}^{*}=\frac{\eta(d-2 \beta c)}{A}+c \\
& g_{C}^{*}=\frac{(d-2 \beta c)\left(\gamma_{i}+\gamma_{r}\right)}{A}
\end{aligned}
$$

式中 $A=4 \beta \eta-\left(\gamma_{i}+\gamma_{r}\right)^{2}>0$ 。由式（9）可知, 市场对产品的基本需求量 $d$ 越大, 则市场定价越加高 于制造成本 $c$; 由式 (10) 可知市场对产品的基本需求量 $d$ 越大, 则企业选择开发产品的绿色属性值越高。 将式 (9) 和（10）带入式（7), 可得集中决策下供应链最大利润如下式:

$\pi^{c}=\frac{\eta(d-2 \beta c)^{2}}{2 A}$

由式 (11) 可知, 集中决策供应链的最大利润总是大于零, 即集中决策下供应链总是可以获得盈利。 


\section{2 分散决策下的模型求解}

分散决策时, 制造商和零售商以自己利润最大化为目标进行独立决策, 零售商决定销售价格, 制造商 决定产品开发的绿色属性和批发价格。该决策过程属于塞头竞争模型, 采用 Stackelberg 博亦来进行分析, 即制造商作为领导者先进行产品开发和批发价格决策，然后零售商作为追随者在制造商行动结果基础上进 行销售价格决策。

首先分析零售商在获知 $w$ 和 $g$ 情况下的最优响应函数，通过分析式 (6) 可知该函数为 $p$ 的凸函数, 当 $p$ 取式 (12) 的数值时获得最大零售商利润。

$$
p_{d}^{*}=\frac{(1-\alpha) d+\gamma_{r} g+\beta w}{2 \beta}
$$

然后将式（12）带入式（5）后得制造商利润表达式如式（13）。

$$
\pi_{i}=(w-c) \frac{(1-\alpha) d+\gamma_{r} g-\beta w}{2}-\frac{\eta g^{2}}{2}+\frac{(1-\alpha) d+\gamma_{r} g+\beta w-2 \beta c}{2 \beta} \frac{3 d(\alpha-1)-\gamma_{r} g-\beta w+2 \gamma_{i} g}{2}
$$

式 (13) 对 $W$ 和 $g$ 求两阶导数分别为 $-1.5 \beta$ 和 $\left(2 \gamma_{i} \gamma_{r}-\gamma_{r}^{2}-\eta\right) /(2 \beta)$, 要使该函数具有极大值, 这两个 表达式均须小于零, 即 $\eta>2 \gamma_{i} \gamma_{r}-\gamma_{r}^{2}$, 且式 (14) 成立。

$$
\eta>\frac{\gamma_{i}^{2}+6 \gamma_{i} \gamma_{r}-3 \gamma_{r}^{2}}{6 \beta}
$$

通常情况下 $\beta 、 \eta$ 要比 $\gamma_{i}$ 和 $\gamma_{r}$ 大，所以在后续研究中假设式（14）总是成立。令 $B=6 \beta \eta-\gamma_{i}^{2}-6 \gamma_{i} \gamma_{r}+3 \gamma_{r}^{2}$, 则式（14）等价于 $B>0$, 此时可以获得分散决策情况下制造商为了获得自身 利润最大化而确定的 $W$ 和 $g$ 最优解分别如式 (15) 和 (16)。

$$
\begin{aligned}
& g_{d}^{*}=\frac{6 \alpha d \gamma_{r}+3 d \gamma_{i}-2 \alpha d \gamma_{i}-4 c \beta \gamma_{i}-3 d \gamma_{r}}{B} \\
& w_{d}^{*}=\frac{1}{\beta B}\left[2 d \alpha\left(\beta \eta-\gamma_{i}^{2}+\gamma_{r}^{2}\right)+d\left(\gamma_{i}^{2}-\gamma_{i} \gamma_{r}\right)+2 c \beta\left(2 \eta \beta-\gamma_{i}^{2}-2 \gamma_{i} \gamma_{r}+\gamma_{r}^{2}\right)\right]
\end{aligned}
$$

此时，零售商制定的最优售价如式（17）。

$$
p_{d}^{*}=\frac{1}{\beta B}\left[2 d \alpha\left(\gamma_{r}^{2}+\gamma_{i} \gamma_{r}-\beta \eta\right)+c \beta\left(2 \beta \eta-\gamma_{i}^{2}-4 \gamma_{i} \gamma_{r}\right)+d\left(3 \beta \eta-2 \gamma_{i} \gamma_{r}\right)\right]
$$

将上述产品开发绿色属性和价格带入式（5）和（6），可得制造商和零售商各自的最大利润，分别记 为 $\pi_{d}^{i^{*}}$ 和 $\pi_{d}^{r^{* *}}$, 供应链整体最大利润记为 $\pi^{d}=\pi_{d}^{i^{*}}+\pi_{d}^{r^{*}}$ 。

\section{3 网购偏好对决策变量影响分析}

通过对模型的详细分析后, 发现网购偏好 $\alpha$ 对系统相关指标具有重要的影响, 本节给出网购偏好 $\alpha$ 对 决策变量的影响规律，证明过程从略。

命题 1 : 分散决策时, 网购偏好 $\alpha$ 与开发产品的绿色属性之间为正相关关系, 与批发价格之间为正相关关系。

命题 2: 分散决策时, 当 $\eta \beta>\gamma_{i} \gamma_{r}+\gamma_{r}^{2}$ 时, 产品零售价与 $\alpha$ 间为负相关关系; 当 $\eta \beta<\gamma_{i} \gamma_{r}+\gamma_{r}^{2}$ 时, 产品零 售价与 $\alpha$ 间为正相关关系; 当 $\eta \beta=\gamma_{i} \gamma_{r}+\gamma_{r}^{2}$ 时，产品零售价与 $\alpha$ 间不相关。 
命题 3: 分散决策时, 网购偏好 $\alpha<\alpha^{*}$ 时, 市场零售价高于批发价, 式中 $\alpha^{*}$ 表达式如下, 且 $0<\alpha^{*}<1$ 。 $\alpha^{*}=\frac{-2 c \eta \beta^{2}+c \beta \gamma_{i}^{2}-c \beta \gamma_{r}^{2}+3 d \eta \beta-d \gamma_{i}^{2}-d \gamma_{i} \gamma_{r}}{d A}$

\section{4 网购偏好对绩效指标的影响分析}

本节给出网购偏好 $\alpha$ 对系统绩效指标的影响规律，证明过程从略。

命题 4: 分散决策时, 网购偏好 $\alpha$ 与直销渠道销售量之间为正相关关系, 与零售渠道销售量之间为负相关关 系。

命题 5: 分散决策时, 当网购偏好 $\alpha<\alpha^{*}$ 时, 网购偏好 $\alpha$ 与制造商利润之间为正相关关系; 当网购偏好 $\alpha>\alpha^{*}$ 时, 网购偏好 $\alpha$ 与制造商利润之间为负相关关系; 当网购偏好 $\alpha_{1}<\alpha<\alpha_{2}$ 时, 制造商能获得利润。式中: $\alpha_{1}=\alpha^{*}-\frac{\sqrt{2 \eta \beta B\left(d^{2}-4 d+4 c \beta\right)}}{2 d A}, \quad \alpha_{2}=\alpha^{*}+\frac{\sqrt{2 \eta \beta B\left(d^{2}-4 d+4 c \beta\right)}}{2 d A}$

由命题 5 可知, $0<\alpha<\alpha^{*}$, 制造商利润随参数 $\alpha$ 增加而增加; $\alpha^{*}<\alpha<1$, 制造商利润随参数 $\alpha$ 增加 而降低。 $0<\alpha<\alpha_{1}$ 或 $\alpha_{2}<\alpha<1$, 制造商不能盈利, 供应链无法运行; $\alpha_{1}<\alpha<\alpha_{2}$, 制造商能够获得盈利。 命题 6: 分散决策时, 网购偏好 $\alpha<\alpha^{*}$ 时, 网购偏好 $\alpha$ 与零售商利润之间为负相关关系, 当 $\alpha \geq \alpha *$ 时, 零 售商的利润为 0 ; 网购偏好 $\alpha<\alpha^{*}$ 时, 网购偏好 $\alpha$ 与供应链利润之间为正相关关系, 当 $\alpha \geq \alpha$ *时, 网购偏 好 $\alpha$ 与供应链利润之间为负相关关系。

命题 7: 当网购偏好在 $\alpha_{1} \leq \alpha \leq \alpha^{*}$ 时, 集中决策供应链利润总是要高于分散决策供应链利润, 且随着 $\alpha$ 接 近 $\alpha^{*}$, 集中决策和分散决策时供应链利润的差别越来越小; 当 $\alpha=\alpha^{*}$ 时, 集中决策和分散决策供应链利润 相等; 网购偏好在 $\alpha^{*} \leq \alpha \leq \alpha_{2}$ 时, 集中决策供应链利润总是要高于分散决策供应链利润, 且随着 $\alpha$ 接近 $\alpha_{2}$, 集中决策和分散决策时供应链利润的差别越来越大。

\section{5 供应链绿色产品开发与渠道分析}

本节分析制造商愿意开发绿色产品、开设直销渠道, 以及零售商愿意加入销售渠道中来的系统条件。

(1) 绿色产品开发的支持条件

在低碳经济时代，不论集中还是分散决策，决策者都希望使得制造商能够开发绿色属性为正的产品， 即 $g>0$ 。

命题 8: 在集中决策情况下, 条件 $d>2 \beta c$ 成立时可使得制造商开发绿色产品; 在分散决策情况下, 条件 $\alpha>\alpha_{m g}$ 成立时可使得制造商开发绿色产品, 其中:

$$
\alpha_{m g}=\frac{4 c \beta \gamma_{i}+3 d \gamma_{r}-3 d \gamma_{i}}{2 d\left(3 \gamma_{r}-\gamma_{i}\right)}
$$

(2) 零售商加入销售渠道的支持条件

分散决策情形下零售商愿意加入销售渠道的条件是其能够获得利润, 即 $p_{d}^{*}>w_{d}^{*}$ 且 $\pi_{d}^{r *}>0$ 。

命题 9: 在分散决策情形下, 当条件 $\alpha<\alpha^{*}$ 成立时, 则 $p_{d}^{*}>w_{d}^{*}$ 且 $\pi_{d}^{r *}>0$, 零售商愿意加入到销售渠道中; 在集中决策情形下, 当条件 $\alpha<\alpha^{*}$ 成立时, 则 $D_{r}>0$, 零售商愿意加入到销售渠道中。 
（3）制造商开设直销渠道的支持条件

分散决策时, 制造商愿意开设直销渠道的条件是能够通过直销渠道销售产品并能够盈利, 即 $D_{i}>0$ 且 $p>c$ 。

命题 10: 分散决策时, 当条件 $\alpha>\alpha_{i}^{*}$ 成立时, 制造商愿意开设直销渠道; 集中决策时, 当条件 $\alpha>\alpha_{c}^{*}$ 成立 时, 制造商愿意开设直销渠道。 $\alpha_{i}^{*}$ 和 $\alpha_{c}^{*}$ 的表达式如下:

$$
\begin{aligned}
& \alpha_{i}^{*}=\frac{2 c \eta \beta^{2}+3 c \beta \gamma_{i}^{2}-4 c \beta \gamma_{i} \gamma_{r}+c \beta \gamma_{r}^{2}+3 d \eta \beta-3 d \gamma_{i}^{2}+d \gamma_{i} \gamma_{r}}{d\left(8 \beta \eta-3 \gamma_{i}^{2}-2 \gamma_{i} \gamma_{r}+\gamma_{r}^{2}\right)} \\
& \alpha_{c}^{*}=\frac{2 c \eta \beta^{2}+c \beta \gamma_{i}^{2}-c \beta \gamma_{r}^{2}+d \eta \beta-d \gamma_{i}^{2}-d \gamma_{i} \gamma_{r}}{d A}
\end{aligned}
$$

命题 11: 分散决策时, 当条件 $\gamma_{r}<3 \gamma_{i}$ 且 $d>2 \beta c$ 成立时, $\alpha_{i}^{*} \geq \alpha_{m g}$ 。

从命题 10 和 11 可知, 在有些条件满足的情况下, 虽然制造商愿意开发绿色产品, 但是当绿色产品属 性比较低, 也即 $\alpha$ 比较小时, 通过直销渠道不能为制造商带来直接利润, 只能通过零售商销售方能获得利 润。

（4）集中决策产品绿色属性高于分散决策的条件

从低碳经济发展的角度看, 集中决策在环境友好性方面要好于分散决策, 总是期望能够通过供应链企 业间的合作实现绿色发展。

命题 12: 当网购偏好在 $\alpha_{m g} \leq \alpha \leq \alpha^{*}$ 时, 供应链集中决策下的产品绿色属性要好于分散决策下的产品绿色 属性，且随着 $\alpha$ 接近 $\alpha^{*}$, 集中决策和分散决策时产品绿色属性之间的差别越来越小。

\section{3. 算例分析}

为了直观地了解双渠道供应链绿色产品开发与销售定价决策中相关参数对决策变量和绩效指标的影 响, 采用算例进行分析。各算例中除了明确指出取值外, 参数 $\left[\alpha, \beta, \gamma_{i}, \gamma_{r}, \eta, c, d\right]$ 取值依次为: [0.48 15810 20050 3000]。

\section{1 网购偏好对系统指标的影响}

根据算例参数, 可以计算出 $\alpha_{m g}$ 为 $0.349, \alpha_{i}^{*}$ 为 $0.437, \alpha^{*}$ 为 0.629 。由这些参数的意义可知, 当 $\alpha$ 取 值在 $\left[{ }^{\alpha_{m g}}, \alpha_{i}^{*}\right]$ 时, 制造商会开发绿色产品, 但是不会开设直销渠道, 产品由零售商销售, 此时的供应链 简称为单渠道两级供应链; 当 $\alpha$ 取值在 $\left[\alpha_{i}^{*}, \alpha^{*}\right]$ 时, 制造商不仅开发绿色产品, 而且会开设直销渠道, 此时的供应链成为双渠道两级供应链; 当 $\alpha$ 取值大于 $\alpha^{*}$ 时, 零售商不会参与到供应链中来, 仅仅是制造商 自行生产和销售, 这种情况本文不进行讨论。因此, 本算例实验中令 $\alpha$ 的取值范围为 $[0.349,0.629]$, 按 照间距 0.028 将 $\alpha$ 取值范围 $[0.349,0.629]$ 划分为 9 等份, 从而获得 $\alpha$ 的 10 个取值, 计算在这 10 个 $\alpha$ 取 值下双渠道供应链最优决策变量取值（图 1) 和系统绩效指标（图 2)。 


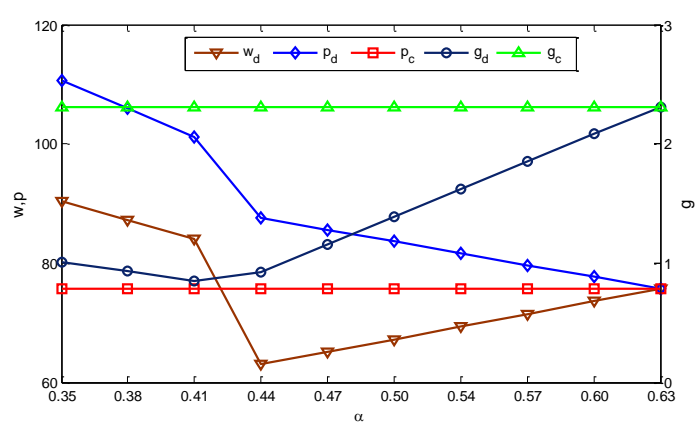

图 1 顾客网购偏好对系统决策变量的影响

图 1 左侧纵坐标对应于系统的决策变量：分散决策下的批发价格 $w_{d}$ 和零售价格 $p_{d}$ 以及集中决策下的 零售价格 $p_{c}$, 右侧纵坐标对应于分散和集中决策下的产品开发绿色属性 $g_{d}$ 和 $g_{c}$ 。从图 1 中可以看出: (1) 集中决策下, 不论 $\alpha$ 如何变化, 供应链的产品绿色属性和零售价格最优取值均维持不变, 其中 $g_{c}$ 为 2.312 , $p_{c}$ 为 75.694 ; (2) 分散决策下, 单渠道两级供应链模式下, 随着 $\alpha$ 的增加, 决策变量 $w_{d} 、 p_{d}$ 和 $g_{d}$ 都逐 步降低; 在双渠道两级供应链模式下, 随着 $\alpha$ 的增加, 批发价格逐步增加而零售价格逐步降低, 当 $\alpha$ 等于 上临界值 0.629 时, 批发价格等于零售价格, 也等于集中决策下的零售价格; (3) 在单渠道两级供应链中, 分散决策零售价格和批发价格均高于集中决策零售价格; (4) 分散决策产品绿色属性总是低于集中决策产 品绿色属性, 但是随着网购偏好的增加, 分散决策产品绿色属性将逼近集中决策产品绿色属性。

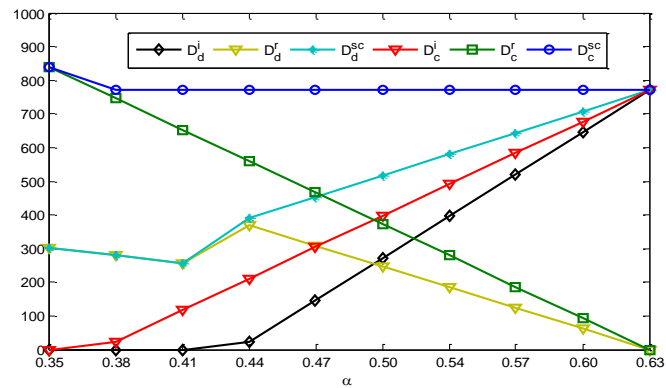

(a)

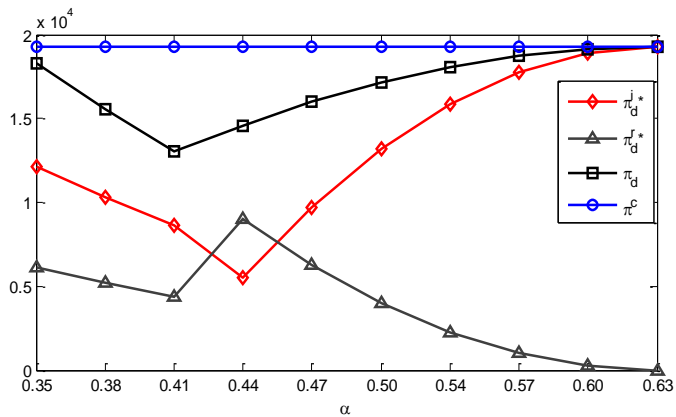

(b)

图 2 顾客网购偏好对系统绩效指标的影响

图 2 中, (a) 展示网购偏好对渠道和供应链销售量的影响，（b）展示网购偏好对渠道和供应链利润的 影响。从图 2（a）可以看出：（1）集中决策下供应链销售量要比分散决策供应链销售量高，但是随着 $\alpha$ 的 增加, 两者差距越来越小, 直至相等; (2) 集中决策下, 随着 $\alpha$ 的增加, 零售商销售量越来越少, 而制造 商直销量越来越多; (3) 分散决策下, 单渠道两级供应链中, 零售商销售量随着 $\alpha$ 的增加而降低; 双渠道 两级供应链中, 零售商销售量随着 $\alpha$ 的增加而降低, 制造商直销销售量随着 $\alpha$ 的增加而增加。从图 2 (b) 可以看出:（1）集中决策供应链总利润要比分散决策供应链总利润高, 但是随着 $\alpha$ 的增加, 两者差距越来 越小, 直至相等; (2) 分散决策下, 单渠道两级供应链中, 零售商、制造商和供应链的利润随着 $\alpha$ 的增加 而降低; 双渠道两级供应链中, 零售商利润随着 $\alpha$ 的增加而降低, 而制造商的利润和供应链的利润随着 $\alpha$ 的增加而提高。 


\section{2 顾客价格敏感系数对系统指标的影响}

顾客对价格的敏感程度影响供应链决策变量和系统绩效, 本小节通过算例分析价格敏感系数 $\beta$ 对供应 链的影响。算例实验中令 $\beta$ 的取值范围为 $[6,24]$, 按照间距 2 将 $\beta$ 取值范围 $[6,24]$ 划分为 9 等份, 从而 获得 $\beta$ 的 10 个取值, 计算在这 10 个 $\beta$ 取值下供应链最优决策变量取值（图 3) 和系统绩效指标（图 4)。

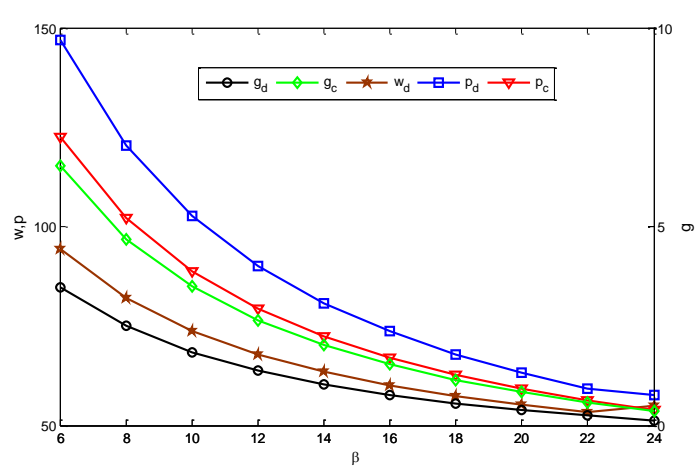

图 3 顾客价格敏感系数对供应链决策变量的影响

图 3 可以看出随着顾客价格敏感系数的逐步增加, 供应链决策变量的变化具有一定规律: (1) 不论是 集中决策还是分散决策, 随着 $\beta$ 的增加, 供应链决策变量都将逐步降低; (2) 分散决策的零售价格总是大 于集中决策的零售价格, 而分散决策的批发价格在顾客对价格敏感程度不高时总是小于集中决策的零售价 格, 但是当 $\beta$ 达到 24 时, 分散决策的批发价格将高于集中决策的零售价格; (3) 集中决策下的产品绿色 属性总是要比分散决策下的绿色属性要高, 尤其在顾客对价格敏感程度较高的时候。

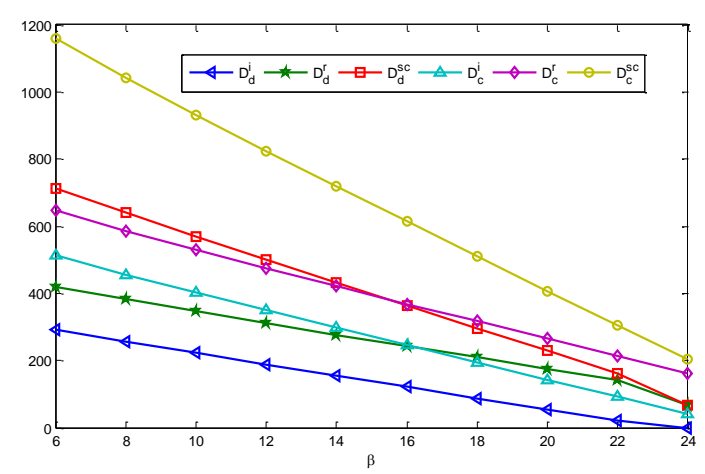

(a)

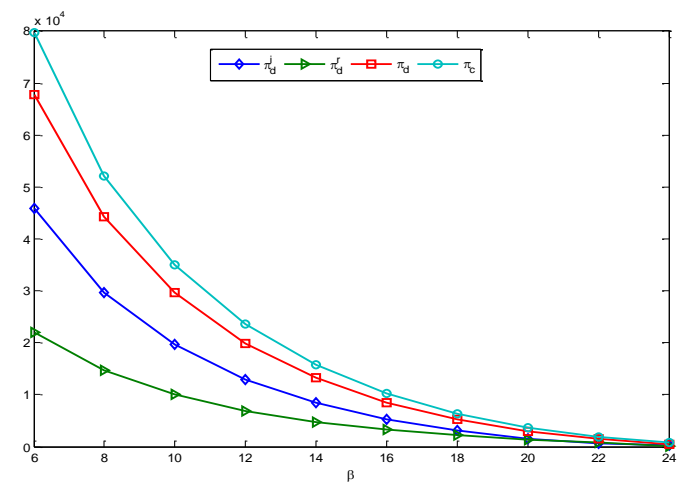

(b)

图 4 顾客价格敏感程度对系统绩效指标的影响

结合图 3 和图 4 反映的规律可以看出, 顾客价格敏感程度越高对供应链整体的利润水平、环保水平都 具有负相关的影响。当顾客对价格非常敏感时, 制造商也会不开设直销渠道, 而仅由零售商销售产品。当 顾客对价格不太敏感时, 集中决策可以获得较高的环保效应。

\section{3 顾客绿色偏好对系统绩效指标的影响}

顾客对产品的绿色偏好程度影响供应链成员的决策和系统绩效, 本节通过算例分析绿色偏好系数 $\gamma_{i}$ 、 $\gamma_{r}$ 的变化对供应链的影响。本算例实验中令 $\gamma_{i} 、 \gamma_{r}$ 在基础数据基础上等比缩放, 缩放系数 $\rho$ 按照步长 0.2 
在区间 $[0.2,2.0]$ 取值, 即 $\rho$ 确定后, 算例试验中对应的直销渠道绿色偏好系数和零售渠道绿色偏好系数 分别为: $\rho \gamma_{i} 、 \rho \gamma_{r}$, 计算在 10 个 $\rho$ 取值下供应链最优决策变量取值和系统绩效指标的变化。通过实验 结果分析后发现, $\rho$ 值的变化对系统决策变量和绩效指标均具有正向的影响, 但是对产品开发的绿色属性 值影响比较大，而对价格变量和利润的影响比较小，具体如图 5（a）和（b）所示。

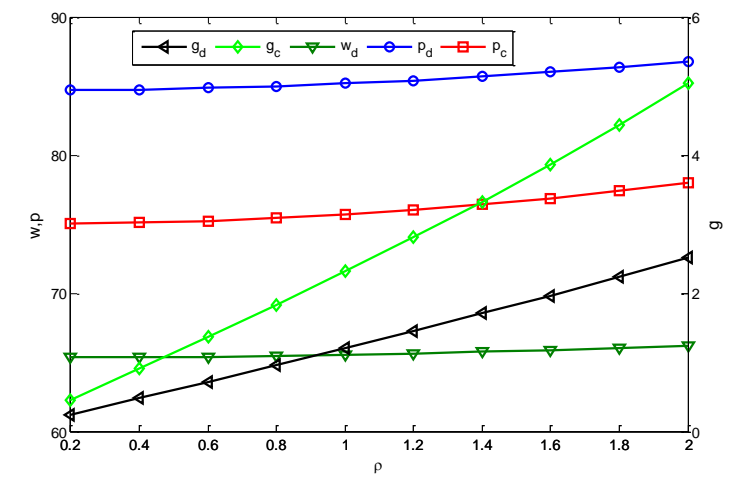

(a)

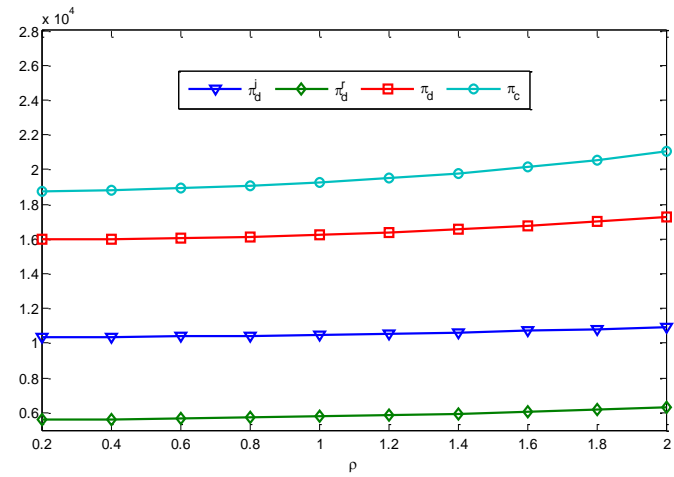

(b)

图 5 顾客绿色偏好对系统绩效指标的影响

图 5 中，（a）展示顾客绿色偏好对系统决策变量的影响，（b）展示顾客绿色偏好对渠道和供应链利润 的影响，而绿色偏好对销售量的影响基本同其对利润的影响，这里从略。从图 6（a）中可以看出：(1) 顾 客绿色偏好对批发价格、零售价格均具有正向影响，但是影响的幅度比较低;（2）三个价格变量满足分散 决策批发价格低于集中决策零售价格、低于分散决策零售价格的规律;（3）顾客绿色偏好对开发产品的绿 色属性具有较强的正向影响, 而且在集中决策下, 随着顾客绿色产品偏好的提高, 供应链对应开发产品绿 色属性的提升幅度要快于分散决策。从图 5（b）中可以看出, 随着顾客绿色偏好的增加, 不论分散决策还 是集中决策，供应链成员及供应链整体的利润都能够有小幅度的提升。可以看出顾客环保意识的增强对绿 色产品的开发和推广具有很强的促进作用, 与此同时可以一定程度上提升供应链成员的利润, 以达到顾客、 企业和环境的多赢状态。

\section{4. 研究结论}

本文研究单制造商和单零售商组成的两级供应链在网络直销渠道和零售商销售渠道并存，市场对绿色 产品具有一定偏好情况下供应链的产品开发和市场定价决策和协调问题，采用博恋论分析方法对集中决策 和分散决策下的供应链决策问题进行优化计算, 并使用数理推理方法导出该类系统所存在的多种内在规律 性。从研究结果可以看出:（1）顾客网购偏好对供应链决策变量和绩效指标具有重要影响, 不仅影响着产 品的绿色属性和销售定价, 而且影响着供应链的结构形态; (2) 顾客对产品绿色偏好程度影响着供应链开 发绿色产品的积极性; （3）集中决策总是要比分散决策下的产品绿色属性要高, 更具有环境友好性; (4) 集中决策供应链整体利润总是要比分散决策供应链整体利润要高。

从本文研究可以看出, 可以通过一系列有效管理举措促进经济的低碳和可持续发展, 例如: (1) 通过 构建品质和服务俱佳的网络购物平台, 促进企业推广网络直销模式, 增强人们网络购物的习惯; (2) 通过 环保、环境和可持续发展资讯或法律法规的宣传教育，使人们认识到所使用产品对地球环境的破坏，进而 提升人们购买绿色产品的偏好; (3) 由政府主导或辅助, 通过产学研合作创新研究产品绿色特征和特性, 
制定相关标准，帮助企业以较低的成本开发具有较高水平的绿色产品，并投入市场；（4）通过各种手段使 供应链企业间能够通过契约实现集中决策;（5）借助 3D 技术实现产品在虚拟场景的呈现，使潜在顾客能 够直接感知产品绿色属性, 增加顾客通过网络采购时的绿色偏好等。这些管理措施将在促进制造商开发具 有更高绿色属性产品的同时，增加供应链的利润。

\section{5. 致谢}

本文研究得到国家自然科学基金项目 71273118 和 71273120 , 江苏省高校哲学社会科学研究项目 2015SJB835，江苏大学高级人才科研启动基金项目 11JDG006 的资助。

\section{Acknowledgement}

The study is supported by National Natural Science Foundation of China(no. 71273118, no. 71273120), Jiangsu provincial philosophy and social science research project (no. 2015SJB835), Excellent Talent Foundation of Jiangsu University(No. 11JDG006) .

\section{参考文献 References}

[1] Niraj Kumar, Ravi P Agrahari, et al. Review of Green Supply Chain Processes[J]. Symposium on Information Control Problems in Manufacturing, 2015. 48(3): 374-381.

[2] Benita M. Beamon. Designing the green supply chain[J]. Logistics Information Management, 1999. 12(4): 332 - 342.

[3] Kuo-Jui Wu, Ching-Jong Liao, et al. Exploring decisive factors in green supply chain practices under uncertainty[J]. International Journal of Production Economics, 2015. 159: 147-157.

[4] Mostafa Zohal, Hamed Soleimani. Developing an ant colony approach for green closed-loop supply chain network design: a case study in gold industry[J]. Journal of Cleaner Production, 2016. 133: 314-337.

[5] Debabrata Ghosh, Janat Shah. Supply chain analysis under green sensitive consumer demand and cost sharing contract[J]. International Journal of Production Economics, 2015. 164: 319-329.

[6] Katsuhiko Takahashi, Takahiko Aoi, et al. Inventory control in a two-echelon dual-channel supply chain with setup of production and delivery[J]. International Journal of Production Economics, 2011. 133(1): 403-415.

[7] S. Panda, N. M. Modak, et al. Pricing and replenishment policies in dual-channel supply chain under continuous unit cost decrease[J]. Applied Mathematics and Computation, 2015. 256: 913-929.

[8] Bo Li, Mengyan Zhu, et al. Pricing policies of a competitive dual-channel green supply chain[J]. Journal of Cleaner Production, 2016. 112, Part 3: 2029-2042. 